Background Male circumcision can protect against sexually transmitted infections, HIV, and urinary tract infections. The procedure is easier to perform during the neonatal period ( $<28$ days), with fewer complications and rarely requiring general anaesthesia. Few studies have estimated the number of circumcisions, or indications for the procedure, in the post-neonatal period (ages 1-18 years). Our objective was to compare these for neonatal and post-neonatal circumcisions.

Methods We analysed MarketScan data, a database of billing claims from commercial health plans. We used circumcision procedural codes to identify all circumcisions in 2010, including neonatal circumcisions of males born to women enrolled in the health plans, and circumcisions of males aged 1-18 years. We assessed reasons for circumcision using diagnostic codes, and stratified the number of circumcisions and associated diagnosis by age. We estimated the neonatal circumcision rate.

Results Overall, 120,994 circumcisions were performed in 2010, with $113,740(94 \%)$ in neonates and 7,254 (6.0\%) in post-neonates. Among post-neonatal circumcisions, $67 \%$ were performed for boys $<3$ years of age and of these $28 \%$ were elective. In contrast, among males 3 years and older, only $8 \%$ were elective. The neonatal circumcision rate was $113,740 / 182,503$ (62\%), and 92\% were elective. Among 16,457 non-elective circumcisions for both neonates and post-neonates, the most frequent indications were phimosis $(92 \%)$, balanitis (3\%), hidden penis $(2 \%)$, chordee $(2 \%)$, and hypospadias $(2 \%)$.

Conclusion Most post-neonatal circumcisions were performed among males $<3$ years, and were 8.6 times higher than circumcisions among males 3 years and older. The large number of elective post-neonatal circumcisions in males $<3$ years suggest that neonatal circumcision might be a missed opportunity for these boys. Delaying elective circumcision results in greater risk for the child, and a more costly procedure. Discussions with parents early in pregnancy might help them make an informed decision about circumcision of their child.

\section{P3.383 MAPPING AND SIZE ESTIMATION OF VULNERABLE YOUTH IN THE WESTERN PROVINCE, SRI LANKA}

doi:10.1136/sextrans-2013-051184.0836

\section{J P h Vidanapathirana, A d Karavita. National STD/AIDS Control Programme,} COLOMBO 10, Sri Lanka

Background All youth in Sri Lanka are not at risk of HIV/AIDS. However, certain socio-economic and behaviour factors are present among youth which make them vulnerable. There are subsets of especially vulnerable youth (EVY) and most-at-risk youth who need to be identified for targeted interventions. Identification of types of subsets and size are essential for interventions. The Objective of this is to identify the subsets of EVY and estimate the size according to the geographical area in the Western Province,

Method Groups of EVY in the western province were identified using participatory approach with broad based consultation of various stakeholders in the public, private and civil society organisation including youth organisations.

Geographic mapping and size estimation was done using the participatory method in group consensus with the key partners. Previous studies, ad hoc surveys, programmatic data from the various organisations including census and statistic data, and experience of the participants were used.

Series of mapping workshops conducted to finalise the locations and size of the population groups identified as EVY. This was done interactively within each group of experts assigned to each study zone. Locations were marked as a point layer manually on Topographic maps. Each point was assigned a definite number relevant to the size.
Results Ten number groups of EVY were identified with total of 47,285 in the Western Province. Final results were generated by using the topographic maps of the Western Province. These maps were then scrutinised carefully in a systematic way to read the locations and the number of the population group marked by group ID. Different point layers for each identified EVY was created on digital topographic maps.

Conclusion Ten groups of EVY were identified according to the size and geographical areas. These data will be using future HIV prevention interventions with different approaches.

\section{P3.384 ESTIMATING THE IMPACT OF COMBINED PREVENTION INTERVENTIONS TARGETING 15-24 YEARS-OLD MEN AND WOMEN IN NYANZA, KENYA}

doi:10.1136/sextrans-2013-051184.0837

'R Alsallaq, ${ }^{1} \mathrm{~J}$ Buttolph, ${ }^{1} \mathrm{C}$ Cleland, ${ }^{2} \mathrm{~T}$ Hallett, 'A Kurth. 'College of Nursing Global, New York University, New York, NY, United States; ${ }^{2}$ mmperial College, London, UK

Background Young males (YMs) and especially young females (YFs) (age 15-24) in sub-Saharan Africa are at a higher risk of HIV infection compared to older adults. HIV testing of young individuals facilitates early identification of their 'HIV +' status, prompt ART initiation, and provision of male circumcision and PrEP. We hypothesise that youth-prioritised combination interventions could have substantial impact on HIV incidence among them and the wider adult population.

Methods We constructed a mathematical model that represented HIV heterosexual transmission in Nyanza, Kenya and used local data to specify cross-generational sex, risk- and age-dependent behaviours, and school attendance. We estimated the impact of leveraging HIV testing and counselling for condom use among Nyanza youth, prompt ART initiation (at CD $4 \leq 350$ cells $/ \mathrm{mm}^{3}$ ) for those newly-found infected, and gender-specific interventions for YMs and YFs. The former reaching $80 \%$ circumcision among ' $\mathrm{HIV}$-' YMs while the later reaching 40\% PrEP coverage among 'HIV-' YFs not attending school and halving the proportion of partnerships that YFs attending school form with $20+$ years-old men.

Results We predict a reduction in HIV incidence over 10 years among youth by $38 \%$ (from $1.6 \%$ /person-years) and adults by $29 \%$ (from 1.2\%/person-years), if the annual testing likelihood for youth increases to $90 \%$ with those newly-found infected increasing condom use by $30 \%$ and initiating ART promptly. The adult incidence is reduced $34 \%$ and $35 \%$ by further male and female specific interventions when applied separately. The full package with all interventions combined would decrease incidence among youth and adults by $59 \%$ and $40 \%$, respectively, and reduce the lifetime HIV risk experienced by YFs by $24 \%$.

Conclusion In populations where young people are at the highest risk of HIV infection, carefully prioritised, gender-specific intervention can have a substantial impact on the risk of infection, both in that group and the overall population.

\section{P3.385 COMPARATIVE EXPERIENCE AND OUTCOMES OF CLINIC STAFF VERSUS INTENSIVE RESEARCHER LED RECRUITMENT TO A SEXUAL HEALTH INTERVENTION IN UK PRIMARY CARE}

doi:10.1136/sextrans-2013-051184.0838

1J A Cassell, ${ }^{2} \mathrm{~J}$ Dodds, ${ }^{1} \mathrm{~S}$ Lanza, ${ }^{3} \mathrm{~N}$ Low, ${ }^{4} \mathrm{~T}$ Roberts, ${ }^{1} \mathrm{H}$ E Smith, ${ }^{5} \mathrm{G}$ Rait. ${ }^{1}$ Brighton and Sussex Medical School, Brighton, UK; '²ueen Mary, University of London, London, UK; ${ }^{3}$ University of Bern, Bern, Switzerland; ${ }^{4}$ University of Birmingham, Birmingham, UK; ${ }^{5}$ University College London, London, UK

Background As part of a national randomised controlled trial, we attempted to recruit young people for Chlamydia testing and 